\title{
Quantifying Wrinkle Features of Thin Membrane Structures
}

\author{
Mindy B. Jacobson ${ }^{*}$ \\ NASA Goddard Space Flight Center, Greenbelt, Maryland, 20771, USA
}

Takashi Iwasa $^{\dagger}$

JAXA, Institute of Space and Astronautical Science, Sagamihara, Kanagawa, Japan

and

M.C. Natori ${ }^{\ddagger}$

JAXA, Institute of Space and Astronautical Science, Sagamihara, Kanagawa, Japan

For future micro-systems utilizing membrane based structures, quantified predictions of wrinkling behavior in terms of amplitude, angle and wavelength are needed to optimize the efficiency and integrity of such structures, as well as their associated control systems. For numerical analyses performed in the past, limitations on the accuracy of membrane distortion simulations have often been related to the assumptions made. This work demonstrates that critical assumptions include: effects of gravity, supposed initial or boundary conditions, and the type of element used to model the membrane. In this work, a $0.2 \mathrm{~m} \times 0.2 \mathrm{~m}$ membrane is treated as a structural material with non-negligible bending stiffness. Finite element modeling is used to simulate wrinkling behavior due to a constant applied in-plane shear load. Membrane thickness, gravity effects, and initial imperfections with respect to flatness were varied in numerous nonlinear analysis cases. Significant findings include notable variations in wrinkle modes for thickness in the range of $50 \mu \mathrm{m}$ to $1000 \mu \mathrm{m}$, which also depend on the presence of an applied gravity field. However, it is revealed that relationships between overall strain energy density and thickness for cases with differing initial conditions are independent of assumed initial conditions. In addition, analysis results indicate that the relationship between wrinkle amplitude scale $(\mathrm{W} / \mathrm{t})$ and structural scale $(L / t)$ is independent of the nonlinear relationship between thickness and stiffness.

$\begin{array}{ll} & \text { Nomenclature } \\ \mathrm{A} & =\text { planar area } \\ \mathrm{F}_{\mathrm{G}} & =\text { force due to gravity } \\ \mathrm{L} & =\text { edge length of membrane } \\ \mathrm{MITC} & =\text { Mixed Interpolation of Tensorial Components, shell element formulation } \\ \mathrm{t} & =\text { membrane thickness } \\ \mathrm{U} & =\text { strain energy density } \\ \mathrm{W} & =\text { peak out-of-plane wrinkle amplitude }\end{array}$

\section{Introduction}

$\mathrm{W}$ ITH the need to develop increasingly more efficient and lighter mechanical and structural systems for spacecraft, many researchers have for decades been experimenting with various types of polymer membranes and working to simulate their behavior. ${ }^{1,2}$ To date, the most detailed simulations have been limited to focusing on

- Aerospace Engineer, Mechanical Systems Analysis \& Simulation Branch, Code 542, Professional AIAA Member.

Research Fellow, JAXA, Section of Spacecraft Engineering, Institute of Space and Astronautical Science, Professional AIAA Member.

${ }^{\ddagger}$ Professor, JAXA, Section of Spacecraft Engineering, Institute of Space and Astronautical Science, Professional AIAA Member. 
macro-scale global distortion and the overall stress field; analysis tools have not been robust enough to quantify wrinkle features such as amplitude, wavelength and orientation angle. In the future, quantified predictions of membrane wrinkling behavior in terms of these three characteristics can be used to optimize the efficiency and integrity of membrane structures and associated control systems.

Historically prominent space-based examples of structural systems employing thin membrane materials include the Echo-1 passive communications satellite, high altitude balloons, and Fresnel lens reflectors. Unlike these systems, future membrane based space structures are likely to require controllability for various precision applications. Because controllability of wrinkled surfaces is a highly complex problem, the current design concept for such structures enforces precision controls on the fabrication processes, rather than on real-time functionality. Examples of some current design concepts of future space-based optics include the James Webb Space Telescope (JWST) sun shield, Constellation-X (CON-X) mirrors, Extreme Universe Space Observatory (EUSO) lenses, and ultra-light precision optics, as shown in Figure 1 (a)-(d), respectively. Each of these structures is highly sensitive to wrinkle patterns in only one dimension, which is an order of magnitude smaller than the other two planar dimensions. Controllability of this dimension and distortion in the out-of-plane direction significantly impacts the function of each system.

Lessons learned from each of these classes of membrane structures can be applied to future controllers and micro-systems only as quantifiable analysis tools become available. The analytical study documented herein includes wrinkle simulations for the basic case of a square membrane fixed at each corner and subjected to inplane shear. Use of the Mixed Interpolation of Tensorial Components (MITC) shell element has enabled this detailed

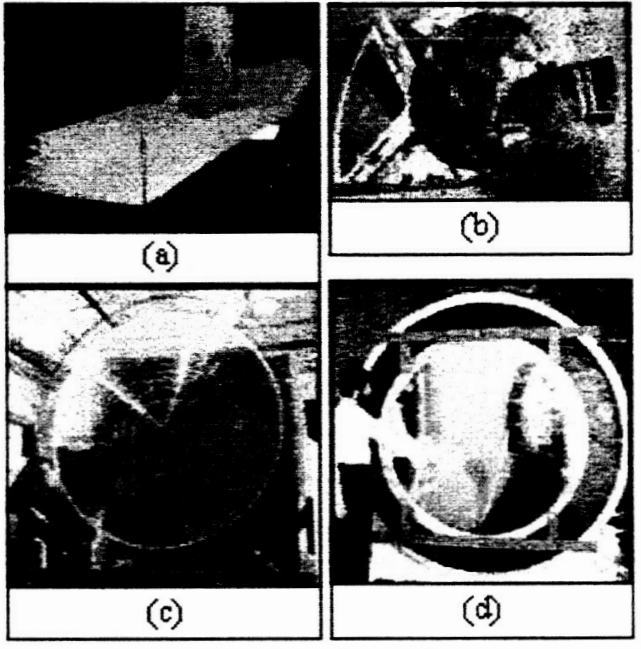

Figure 1. Space-based membrane structures: (a) JWST sunshield concept, (b) CON-X mirror, (c) EUSO lenses, (d) ultra-light precision optics

quantitative study for reasons discussed in the following section. The subsequent section describes numerous nonlinear analysis cases considered, in which membrane thickness, gravity effects, and initial imperfections with respect to flatness were varied. Simulation results are presented and quantified in terms of wrinkle amplitude, orientation and wavelength.

Results of this work are significant as a tool for: (1) assessing numerical analysis sensitivities in preparation for data correlation, and (2) developing alternative tools for analyzing membrane wrinkling behavior predicted by experiments or finite element methods. ${ }^{3}$

\section{Wrinkling Theory}

Although a complete history of the development of theories for explaining wrinkling behavior are beyond the scope of this paper, three theories traditionally used to study and simulate wrinkled membranes are briefly summarized here.

First, Tension Field Theory (TFT) mathematically describes wrinkling in terms of solid mechanics. ${ }^{4}$ It asserts that membranes can be described as structures of sufficiently little thickness such that they have zero bending stiffness. As a result, they have no capacity to support compressive load or carry compressive stress.

Plate Theory is assumed when considering structural responses that are dominated by in-plane structural stiffness. Out-of-plane, or transverse loads are considered negligible by comparison. Elements treated according to this theory are not expected to undergo significant amounts of bending, although they do have significant thickness.

Classic Shell Theory is assumed when a thin structural element is loaded both in-plane and out-of-plane, and bending is expected. The theory includes non-negligible bending stiffness and can be used for considering nonlinear deformation, or strain. Further, it considers that the shell is an inextensible surface and is modeled at the mid-plane of that surface - therefore, transverse stresses through the thickness of a surface are not captured.

For elastic materials, such as membranes considered in this work, inextensibility is a fundamental principle stating that the length of a distorted path cannot exceed the length of an undistorted path of the same structure. In other words, geometric compatibility between initial and final states must be preserved. For wrinkling phenomena, this principle relates to the transverse direction. Hence, Plate Theory is insufficient for characterizing wrinkling behavior. Classic Shell Theory is sufficient only when formulations including transverse shear stresses are 
employed. Such formulations include, for example, Discrete Kirchhoff Shells and Mixed Interpolation of Tensorial Components (MITC) shells.

The MiTC sheli element was developed by K. J. Bathe and E. N. Dvorkin to combine the plate and sheil theories in finite element analyses. 5,6 The stress and strain tensors at each element node are interpolated along each edge of the element, as well as through the element thickness. The interpolation scheme used makes this element more resistant to shear locking than other shell elements. Altogether, the characteristics of the MITC shell element allow them to capture true strain energy density levels. This is critical, given that wrinkling is best described as the minimum energy response for accommodating in-plane strain while also satisfying the inextensibility principle in the transverse direction.

Mathematically, wrinkles have been described in terms of both TFT and shell theory. For the purposes of this study, to be consistent with MITC element formulation, the principles of shell theory are used to characterize wrinkle states, as described in the following Table 1.

\section{Table 1: Wrinkle State Criteria Defined by Principai Stresses}

\begin{tabular}{cl}
\hline Taut & $\sigma_{11}>0$ and $\sigma_{22}>0$ \\
Wrinkled & $\sigma_{11}>0$ and $\sigma_{22} \leq 0$ \\
Slack & $\sigma_{11} \leq 0$ and $\sigma_{22} \leq 0$ \\
\hline
\end{tabular}

\section{Analysis}

\section{A. Method}

The computational tool used to perform these simulations is a Finite Element Analysis Program: Personal Version (FEAPpv). ${ }^{x, 9,10}$ The 4-node MITC shell element was added by authors to the element library. The Newton and modified Newton methods are used to compute nonlinear analysis solutions.

\section{B. Model}

The $0.2 \mathrm{~m} \times 0.2 \mathrm{~m}$ membrane finite element model is shown in Figure 2. The mesh was discretized with regard to optimal aspect ratio for best performance of the 4-node MITC shell element. " Computation time is of secondary importance in this study; the model consists of 10000 elements $\left(0.002 \mathrm{~m}^{2}\right.$ each) and 10201 nodes.

The membrane is constrained at 3 of 4 comers in all degrees of freedom except out of plane rotation. At the fourth corner of the membrane, a small displacement is applied in the direction of the $+X$ axis $(\delta x=0.001 \mathrm{~m})$ and constraints are applied against translation in the $Y$ and $Z$ directions, as well as against rotation about the $X$ and $Y$ axes.

The magnitude of applied displacement was set with consideration for the assumption that this analytical study

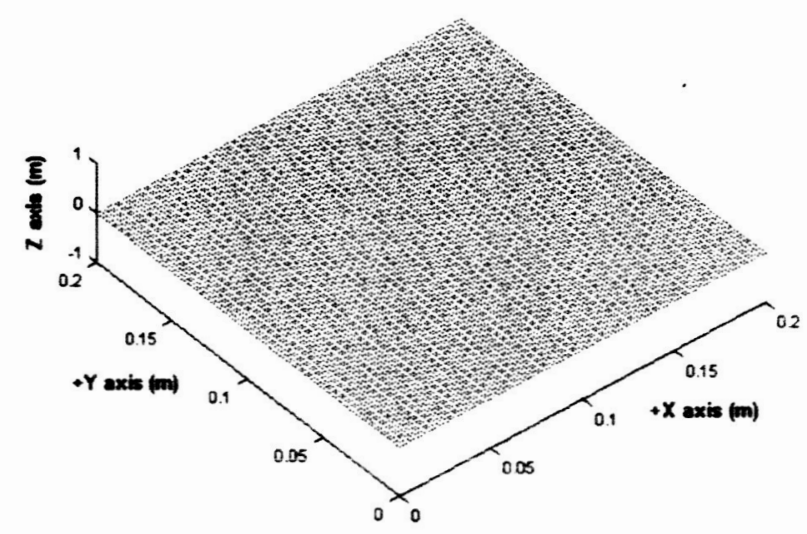

Figure 2: Finite Element Model is based on linear elastic behavior of the membrane. Resultant global stress levels are not to exceed the $5 \%$ yield point for H-type Kapton; refer to Table 2 for assumed material properties.

Table 2: H-type Kapton Material Properties

\begin{tabular}{ccc}
\hline Property & Value & Unit \\
\hline Tensile Strength, Ultimate & 243.04 & $\mathrm{MPa}$ \\
Yield Point (3\% offset) & 95.06 & $\mathrm{MPa}$ \\
Yield Point (5\% offset) & 123.48 & $\mathrm{MPa}$ \\
Elongation, Ultimate & 80 & $\%$ \\
Young's Modulus, E & 2959.6 & $\mathrm{MPa}$ \\
Poisson's Ratio, $v$ & 0.34 & \\
Density, $\rho$ & 1.42 & $\mathrm{~g} / \mathrm{cm}^{3}$ \\
\hline \hline
\end{tabular}




\section{Cases}

Parameters discussed in the previous section are held constant for all numerical simulations. Each analysis case performed in this study was derived based on the need to systematically evaluate effects of additional analytical variables. Table 3 lists the variable parameters treated.

For each thickness, geometrically nonlinear analyses were performed in order to assess the wrinkling behavior both with and without the gravity effect, which is assumed perpendicular to the plane of the membrane. For the cases including this effect, membrane sag due to gravity is taken to be the initial distorted shape prior to applying the shear

Table 3: Pârameterss Varied for Analytical Study

\begin{tabular}{|c|c|c|}
\hline Parameter & Values & Units \\
\hline Thickness, $\mathrm{t}$ & $\begin{array}{c}50,125,150,175 \\
300,500,1000\end{array}$ & $\mu \mathrm{m}$ \\
\hline Gravity & 0 or $(\rho \cdot A \cdot t) \cdot G$ & $\mathrm{M}-\mathrm{N}$ \\
\hline $\begin{array}{l}\text { Initial Flatness } \\
\text { Imperfection }\end{array}$ & $\begin{array}{c}-10 \% \mathrm{t} \leq \mathrm{NRN} \leq \\
+10 \% \mathrm{t}\end{array}$ & $\mu \mathrm{m}$ \\
\hline
\end{tabular}
load (as an enforced displacement, $\delta x=0.001 \mathrm{~m}$ ).

For cases not including the gravity effect, the initial flatness of the membrane is assumed to not be perfect. That is, in reality, no square membrane surface can be considered perfectly flat when it is suspended at each of its 4 corners. In this study, the initial deformed shape (or, flatness imperfection) is determined in one of two ways. The first method is to apply random values of out-of-plane distortion at each node. These values are $\pm 10 \%$ of the membrane thickness and are taken from a normal random number (NRN) generator, and they are applied as initial

Table 4: Description of Analysis Cases

\begin{tabular}{|c|c|c|c|c|}
\hline Case & $\begin{array}{l}\text { Applied } \\
\text { Load }\end{array}$ & Initial Condition & $\begin{array}{l}\text { Boundary } \\
\text { Condition }\end{array}$ & Illustration \\
\hline "Self-Weight" & $\begin{array}{c}\text { Effective } \\
\text { Gravity } \\
\text { Force at } \\
\text { Each Node }\end{array}$ & None & $\begin{array}{l}\text { 5DOF Constrained } \\
\text { at All } 4 \text { comers }\end{array}$ & \\
\hline "Gravity" & $\begin{array}{c}\delta \mathrm{x}=0.001 \mathrm{~m} \\
\text { at one } \\
\text { corner } \\
(\mathrm{X}=0.2 \mathrm{~m} \\
\mathrm{Y}=0.0 \mathrm{~m})\end{array}$ & $\begin{array}{l}\text { Restart of Self- } \\
\text { Weight Case }\end{array}$ & $\begin{array}{l}\text { 5DOF Constrained } \\
\text { at } 3 / 4 \text { corners; } \\
\text { 4DOF constrained } \\
\text { at corner of } \\
\text { applied load }\end{array}$ & \\
\hline $\begin{array}{l}\text { "No Gravity } \\
\text { NRN" }\end{array}$ & \multirow{2}{*}{$\begin{array}{c}\delta \mathrm{x}=0.001 \mathrm{~m} \\
\quad \text { at one } \\
\text { corner } \\
(\mathrm{X}=0.2 \mathrm{~m} \\
\mathrm{Y}=0.0 \mathrm{~m})\end{array}$} & $\begin{array}{c}\text { Flatness } \\
\text { Imperfection due } \\
\text { to random } \\
\text { distribution of } \\
\text { distortions }(\delta z)\end{array}$ & \multirow{2}{*}{$\begin{array}{l}\text { 5DOF Constrained } \\
\text { at } 3 / 4 \text { corners; } \\
\text { 4DOF constrained } \\
\text { at comer of } \\
\text { applied load }\end{array}$} & \\
\hline $\begin{array}{l}\text { "No Gravity } \\
\text { S-W" }\end{array}$ & & $\begin{array}{l}\text { Sag shape due to } \\
\text { Self-Weight } \\
\text { analysis }\end{array}$ & & \\
\hline
\end{tabular}


conditions. Alternatively, the initial deformation is assumed to correspond to the sag shape due to gravity, but applied as a geometric initial condition, rather than taken as the result of an applied load.

Table 4 summarizes each of the analysis cases considered in this study. Ail cases, but the first ("Self-Weight"), are considered to be wrinkling analysis cases for which a detailed study of the results has been conducted. The "Self-Weight" case is taken to be a baseline for comparing the quantified effects due to gravity simulated in the other three wrinkling cases. Note that each membrane thickness given in Table 3 is considered for all cases.

\section{Results}

\section{A. Self-Weight Case}

The purpose of this case is to simulate the static effect of a (1G) gravity field on a square membrane held fixed (5DOF) at all four corners. The gravity field is simulated by computing an effective gravity force $\left(F_{G}\right)$ for each membrane thickness, which is evenly distributed over the entire area (A) and applied discretely at each node.

Figure 3 illustrates the resultant deformed shape that is characteristic for each membrane thickness, from $50 \mu \mathrm{m}$ to $1000 \mu \mathrm{m}$. Note that the shape of any cross section profile is not a catenary.

For comparison with the three wrinkling analysis cases, total strain energy density (U) for each membrane thickness is presented in Figure 4; a nondimensional structural scale $(\mathrm{L} / \mathrm{t})$ parameter is given along the abscissa.

It is clear that $U$ rapidly decreases with thickness. The rate of decrease is related to the rate of decrease in membrane stiffness, which is a compound function of both $t$ and $t^{3}$ for in-plane and bending stiffness, respectively. Overall work required to distort the membrane is significantly less for small thickness than for large; therefore, this relationship is expected.

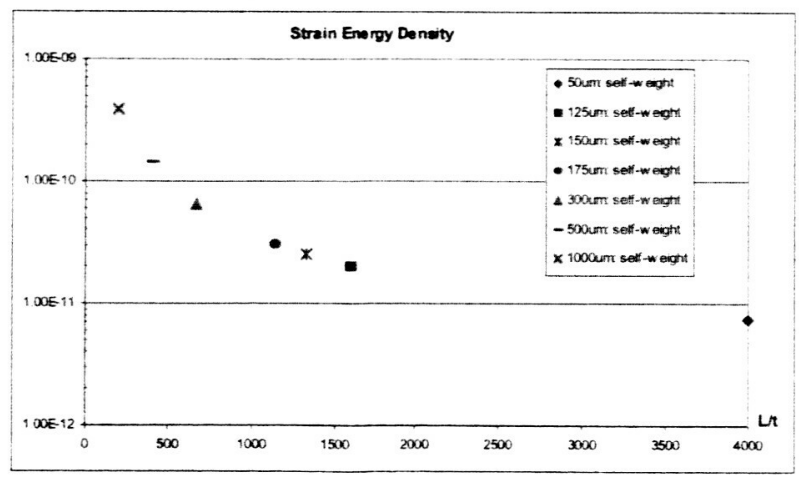

Figure 4: Strain Energy Density Due to Gravity Sag

\section{B. Gravity Case}

The solution of this case is obtained from a nonlinear solution re-start of the "Self-Weight" case while applying the corner shear force. In this sense, the simulation may be considered to be the result of an initial applied load, or force. (This is contrary to the "No Gravity S-W" case, described below, in which the gravity effect is modeled as a geometric initial condition, or distortion.)

Although simulations for each thickness given in Table 3 were performed, for brevity only the deformed shapes for $\mathrm{t}=50,175,300$, and $1000 \mu \mathrm{m}$ are illustrated in Figure 5. Simulation results indicate that only when $\mathrm{t}<500 \mu \mathrm{m}$, maximum out-of-plane distortions ( $\delta z$, in either the $+Z$ or $-Z$ direction) decrease as thickness decreases. However, there is no clear thickness dependent trend for maximum stress levels, which occur at the point of application of the corner shear load; the difference between maximum and minimum values is only $5.7 \%$. 
Element-by-element analysis of wrinkle state, as defined in Table 1, for each membrane thickness has revealed that for $\hat{t}>300 \mu \mathrm{m}$, wrinkle criteria based on major principal stresses does not accurately characterize membrane distortion because some regions do carry some level of compressive stress without buckling due to sufficiently high levels of bending stiffness. This may be confirmed by examining the deformation modes given in Figure 5; true wrinkling behavior (a result of localized buckling) is dominant only when $\mathrm{t} \leq 300 \mu \mathrm{m}$.

\section{No Gravity NRN Case}

This analysis case was prepared by enforcing, as a geometric initial condition, small out-of-plane $(\delta z)$ distortions at each node location. The magnitude of these distortions is not greater than $10 \%$ of the membrane thickness, and they were taken from a normal random number (NRN) generator.

This initial condition was chosen in order to induce

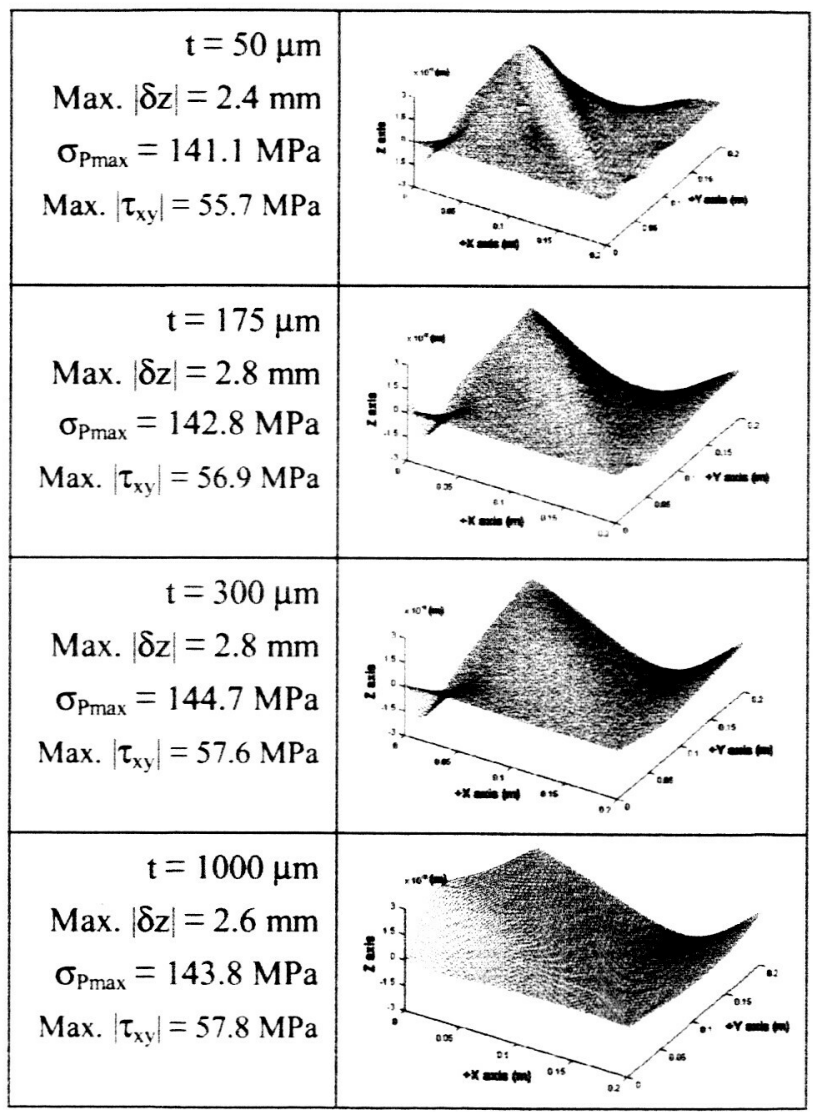

Figure 6: Wrinkled Membranes Due to Applied Corner Shear Load, Including Initial NRN Imperfection

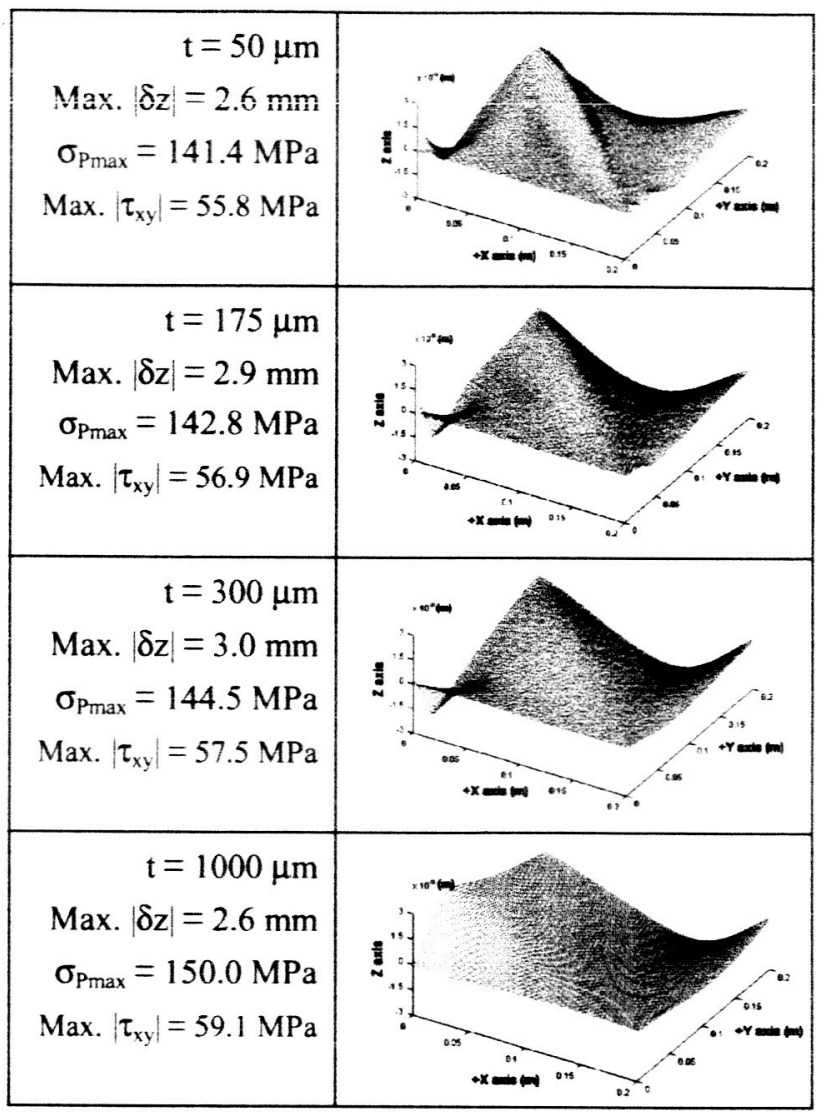

Figure 5: Wrinkled Membranes Due to Applied Corner Shear Load, Including Gravity Field

a perturbation in the flatness of the membrane - in the absence of a gravity effect. The perturbation is necessary because the assumption of a perfectly flat surface is an inherently stable system. The nature of the NRN displacement initial condition allows for the computation of a solution based on imperfect bifurcation, meaning that no single eigenvector trajectory is followed through the solution sequence.

As in Figure 5 for the "Gravity" case, Figure 6 illustrates wrinkled membranes only for $t=50,175$, 300 , and $1000 \mu \mathrm{m}$. A quantitative comparison of the stresses and deformations associated with these results and those given in Figure 5 reveals no significant differences. However, there are obvious qualitative differences as thickness decreases.

Therefore, in order to truly characterize wrinkling behavior it is necessary to quantify wrinkle orientation in addition to amplitude. This has been done, for this study, in a rudimentary way by plotting maximum and minimum principle stresses at a constant cross section in the membrane, for each thickness as shown in Figure 7 . The most significant detail to notice from this

This topic is expanded and thoroughly treated in a separate study entitled "A Spectrum Evaluation Method for Wrinkled Membranes Using Two-Dimensional Fast Fourier Transform" by T. Iwasa, et. al. ${ }^{3}$ 
comparison is that minor principle stresses tend to reverse, and with greater frequency, as thickness decreases. This

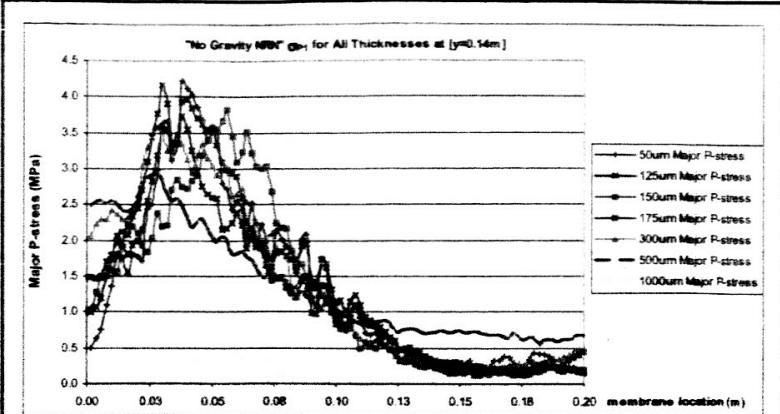

(a) Major Principal Stresses, $\sigma_{11}$

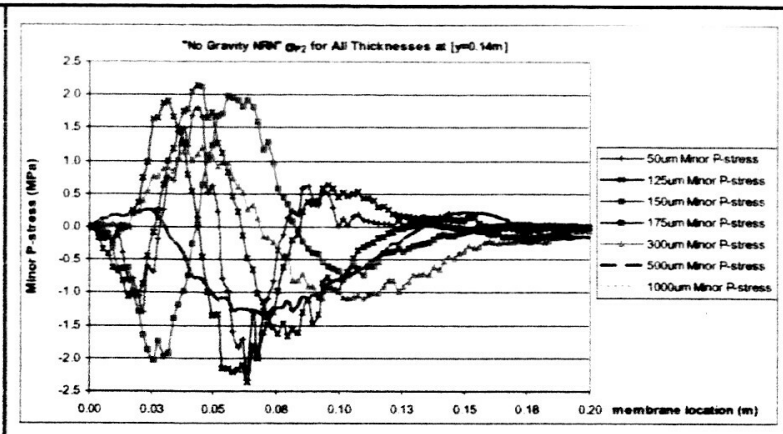

(b) Minor Principal Stresses, $\sigma_{22}$

Figure 7: $\sigma_{11}$ and $\sigma_{22}$ Along Membrane Cross Section at $Y=0.14 \mathrm{~m}$, Including Initial NRN Imperfection is the cause of increasingly numerous and skewed wrinkles when thickness is less than $300 \mu \mathrm{m}$.

\section{No Gravity S-W Case}

This wrinkling case differs from those presented in the previous two sections in that the assumed initial condition is determined by the appropriate degree of gravity sag deformation, depending on thickness, that was computed in the "Self-Weight" case. This is unlike the "Gravity" analysis case because the initial condition is applied as a geometric constraint, rather than as a distributed applied load $\left(\mathrm{F}_{\mathrm{G}}\right)$.

Figure 8 gives analysis results similar to those reported in Figures 5 and 6, for $t=50,175,300$, and $1000 \mu \mathrm{m}$. These results reveal similar trends with

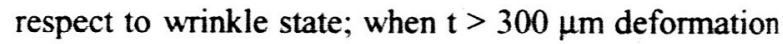
may not be accurately characterized by the term "wrinkled", according to the state definitions given in Table 1. In addition, stress levels and out-of-plane deformation are not significantly different than the other two wrinkling cases.

However, the deformed state when $t=50 \mu \mathrm{m}$ is different than the previous two cases. In addition, differences are discovered when carefully analyzing membrane deformation at constant cross sections using the wrinkle scale parameter $(\mathrm{W} / \mathrm{t})$. This is discussed in greater detail in the following section.

\section{Discussion}

In order to visualize how wrinkling behavior is effected by the different initial conditions it is beneficial to examine cross section plots at constant thickness for each analysis case. The non-dimensional wrinkle scale parameter $\mathrm{W} / \mathrm{t}$ is plotted versus structural scale $\mathrm{L} / \mathrm{t}$, where $\mathrm{W}=$ peak out-of-plane displacement and $\mathrm{L}=$ edge length. For illustration, here, the cross section plots for $\mathrm{t}=50,175,300$, and $1000 \mu \mathrm{m}$ at $\mathrm{y}=0.14 \mathrm{~m}$ are shown in Figure 9.

A detailed examination of such plots for each membrane thickness reveals that deformation mode is independent of initial conditions when $\mathrm{t} \leq 150 \mu \mathrm{m}$. Also, for any thickness, the deformation modes for "Gravity" and "No Gravity

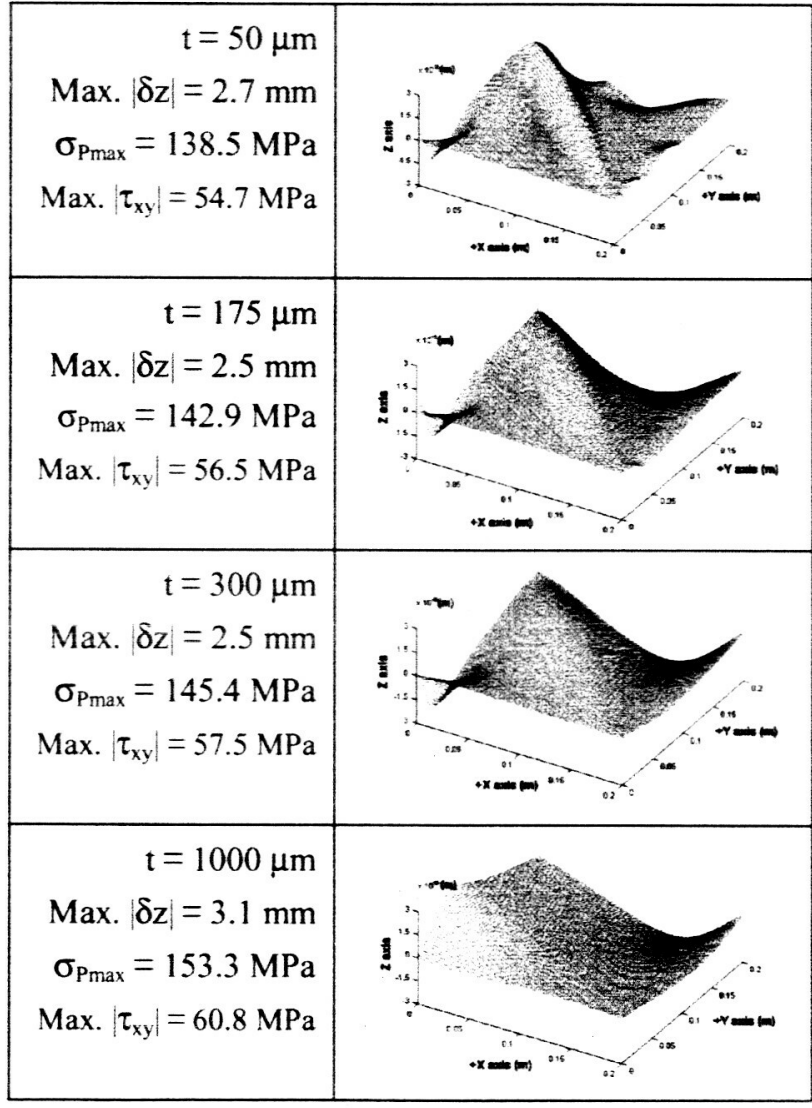

Figure 8: Wrinkled Membranes Due to Applied Corner Shear Load, Including Initial Self-Weight Gravity Sag Shape 


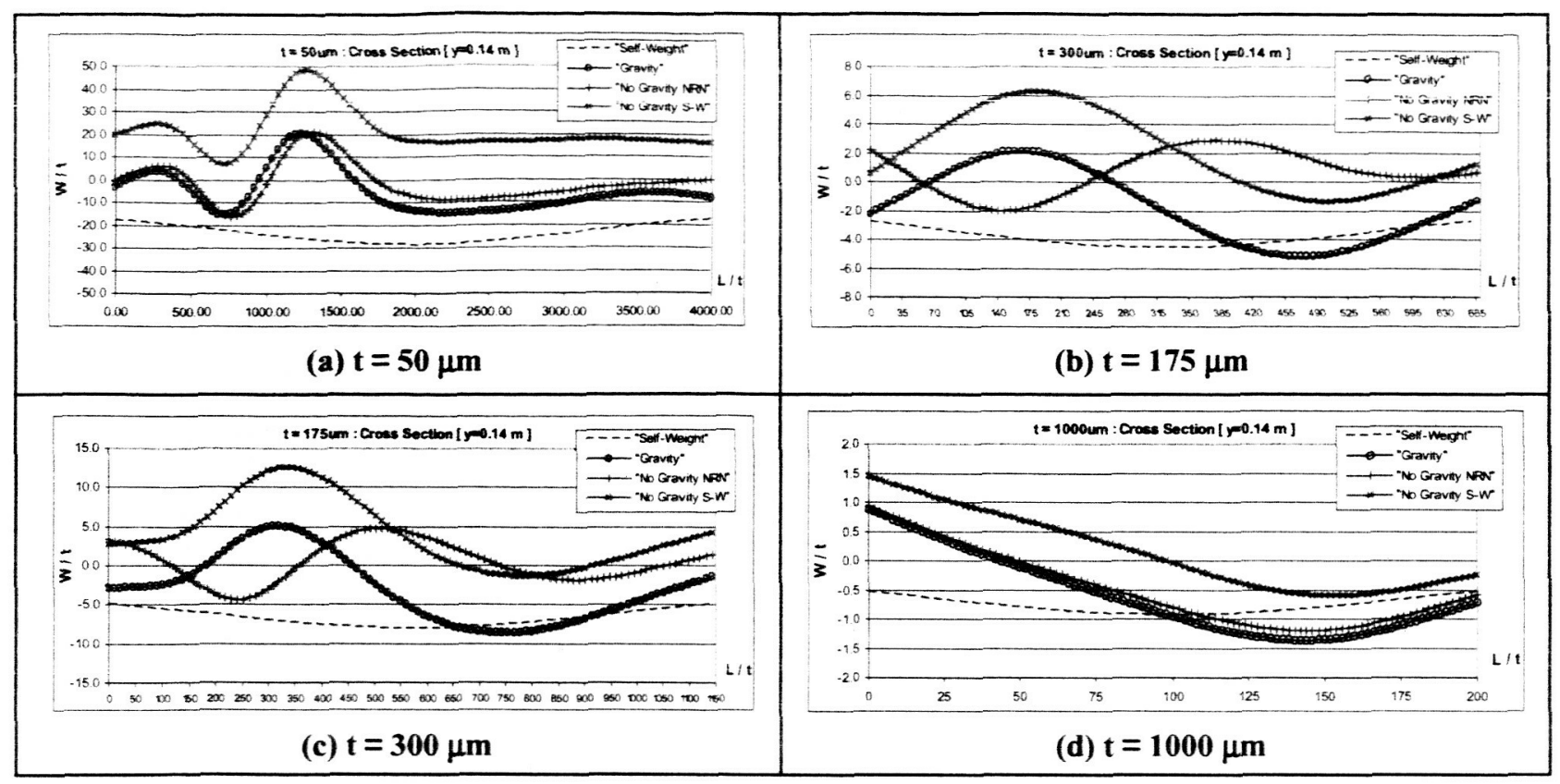

Figure 9: (W/t) Along Membrane Cross Section at $Y=0.14 \mathrm{~m}$

S-W" are the same, but offset by the initial $\delta z$ offset due to the gravity sag shape. The disparity between these two deformation modes and that for the "No Gravity NRN" case becomes noticeable when $175 \mu \mathrm{m} \leq \mathrm{t}<1000 \mu \mathrm{m}$ and a phase shift between wrinkle peaks becomes apparent. But, when $t=1000 \mu \mathrm{m}$, deformation mode is again independent of initial conditions. Another prominent trend to notice is that wavelength increases with thickness, regardless of assumed initial conditions.

Figure 10 illustrates the relationship between the wrinkle $(\mathrm{W} / \mathrm{t})$ and structural $(\mathrm{L} / \mathrm{t})$ scale parameters. (Recall that wrinkle scale is defined by the peak out-ofplane displacement.) It is important to note that this relationship is linear for the "Gravity" case when $\mathrm{t}<150 \mu \mathrm{m}$. This indicates that, for certain structural scales, analysis results may be scaled for correlation with ground-based experimental data - but only for square geometries. That is, previous research studying similar behavior but for a circular membrane has revealed that the same relationship is not linear. ${ }^{12}$ Rather, it increases nonlinearly towards a constant value as $\mathrm{L} / \mathrm{t}$ increases. So, the relationship between wrinkle scale and structural scale depends on geometry, not initial conditions.

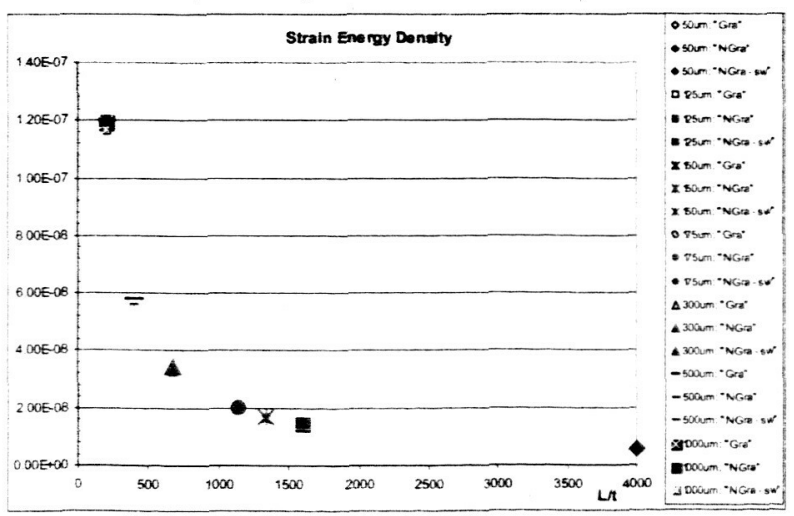

Figure 11: Strain Energy Density versus (L/t) for All Wrinkling Cases

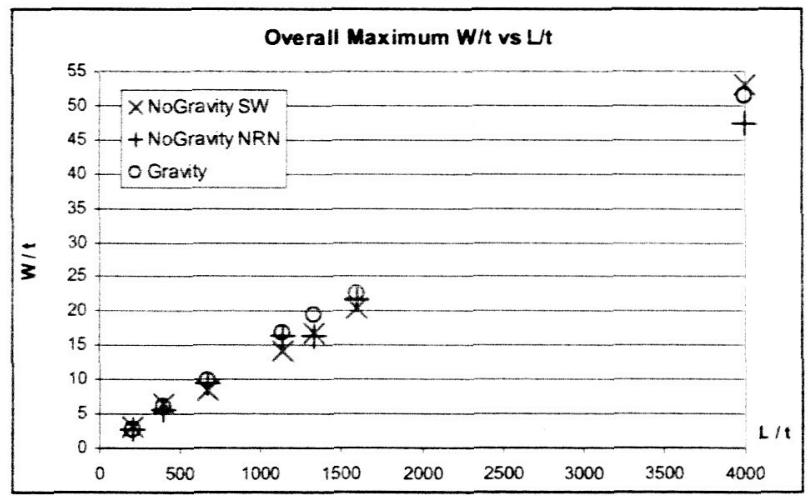

Figure 10: Overall Maximum (W/t) versus (L/t)

Plotting total strain energy density versus structural scale reveals the same relationship for all three wrinkling analysis cases as was revealed for the "Self-Weight" case. Refer to Figure 11, which shows that volumetric strain energy decreases rapidly with thickness and approaches a constant value. Therefore, the minimum energy response for accommodating in-plane strain (in the form of wrinkles) approaches a constant as thickness becomes small. However, as previously noted, when $t \geq 500 \mu \mathrm{m}$ wrinkling is not an accurate description of the deformation due to in-plane shear.

With respect to strain energy density, it is also interesting to note that the ratio of results for the "Gravity" 
wrinkling case and the baseline "Self-Weight" cases increases rapidly and approaches a constant value as thickness decreases (refer to Figure 12).

Additional analyses have revealed that the ratio of strain energy density in the "Gravity" case to that for either of the other two wrinkling cases is $1.0 \pm 5 \%$, regardless of thickness. Therefore, initial conditions do not significantly impact the energy state of a wrinkled membrane. Other parameters relating wrinkle amplitude to orientation are necessary to fully quantify and qualify wrinkle behavior.

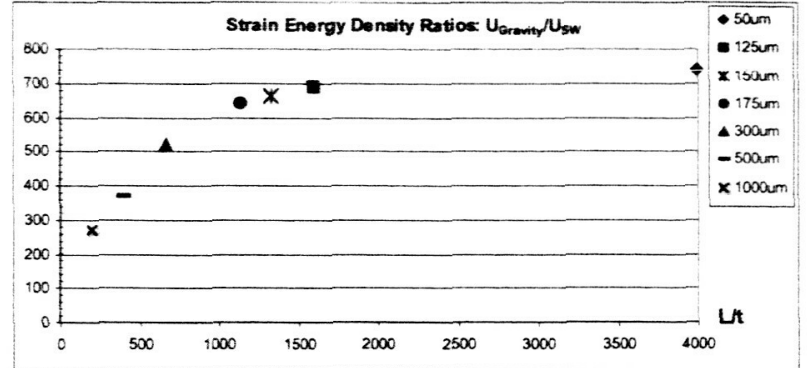

Figure 12: Strain Energy Density Ratio: $\boldsymbol{U}_{\text {Gravity }} / \mathbf{U}_{\text {Self-Weight }}$

\section{Summary}

A systematic analytical study of the affects of thickness, gravity, and initial conditions on membrane wrinkling behavior has been presented. The universally applied static load in this study is in-plane shear, applied at one corner of a square membrane held fixed at the remaining three corners. This study is unlike others previously conducted on wrinkling behavior because of the geometry and numerical methods used to simulate behavior.

Detailed analysis of the simulation results for three different wrinkling cases reveal important relationships between deformation and thickness, as well as initial conditions:

- As thickness decreases from the maximum considered in this study $(1000 \mu \mathrm{m})$, the global wrinkling behavior is sensitive to initial conditions only when thickness is less than $150 \mu \mathrm{m}$.

- As thickness increases, greater than $300 \mu \mathrm{m}$, membrane deformation may no longer be mathematically characterized as wrinkling.

- Overall stress and strain levels are not significantly effected by assumed initial conditions, though wrinkle orientation is.

- Wrinkle wavelength increases with thickness for any assumed initial condition.

- By plotting wrinkle scale with respect to structural scale, it is shown that wrinkle scale is independent of the nonlinear relationship between thickness and stiffness.

- The relationship between wrinkle scale and structural scale is linear when $\mathrm{t}<150 \mu \mathrm{m}$ for the "Gravity" case in this study.

- The ratio of overall strain energy density in the "Gravity" case to that for either of the other two wrinkling cases is approximately 1.0 .

All of these results may be useful for correlating test data from a ground-based experiment. In addition, they reveal the fact that quantifying wrinkle behavior due to in-plane shear is a laborious and time-consuming task when utilizing finite element simulations. Because knowledge of precise wrinkle characteristics such as orientation and wavelength may be necessary for optical applications of membrane structures, this analytical study may be useful as the background for developing alternative wrinkling analysis tools capable of predicting such details outright.

\section{Acknowledgments}

We thank H. Noguchi for providing support with respect to incorporating the MITC shell element in to FEAPpv.

\section{References}

'Stein, M. and Hedgepeth, J.M. "Analysis of Partly Wrinkled Membranes." NASA Technical Note. NASA-TND813, 1961.

${ }^{2}$ Mikulas, M. M. Jr., "Behavior of a Flat Stretched Membrane Wrinkled By the Rotation of an Attached Hub," NASA Technical Note. NASA-TN-D-2456, 1964.

${ }^{3}$ Iwasa, T., Jacobson, M., Natori, M.C., "A Spectrum Evaluation Method for Wrinkled Membranes Using TwoDimensional Fast Fourier Transform," 45th AIAA/ASME/ASCE/AHS/ASC Structures, Structural Dynamics and Materials Conference Proceedings, 2003 (to be published).

${ }^{4}$ Reissner, E., "On Tension Field Theory," Proc.V Int. Cong. Appl. Mech., 1938, pp. 88-92. 
5 Bathe, K.J. and Dvorkin, E.N., "Short Communication: A Four-node Plate Bending Element Based on Mindlin/Reissner Plate Theory and A Mixed Interpolation," Int. J Num. Meth. Engr., Vol. 21, 1985, pp. $367-383$.

${ }^{6}$ Bathe, K.J. and Dvorkin, E.N., "A Formulation of General Shell Elements - The Use of Mixed interpolation of Tensorial Components," Int. J Num. Meth. Engr., Vol. 22, 1986, pp. 697-722.

${ }^{7}$ Bathe, K.J., Brezzi, F., and Fortin, M., "Mixed-interpolation Elements for Reissner-Mindlin Plates," Int. J Num. Meth. Engr, Vol. 28, 1989, pp. 1787-1801.

${ }^{8}$ Taylor, R.L. FEAP - A Finite Element Analysis Program Version 7.4 Programmer Manual. University of California Press, Berkeley, 2001.

${ }^{9}$ Taylor, R.L. FEAPpv - A Finite Element Analysis Program, Personal Version 1.0 User Manual. University of California Press, Berkeley, 2001.

${ }^{10}$ Taylor, R.L. FEAP - A Finite Element Analysis Program Version 7.4 Theory Manual. University of California Press, Berkeley, 2002.

11 Chapelle, D. and Bathe, K.J., "Fundamental Considerations for the Finite Element Analysis of Shell Structures," Computers \& Structures, Vol. 66.1, 1998, pp. 19-36.

${ }^{12}$ Iwasa, T., Natori, M.C., Noguchi, H. and Higuchi, K., "Geometrically Nonlinear Analysis on Wrinkling Phenomena of a Circular Membrane," Research Report on Membrane Structures, Vol. 16, 2002, pp. 7-14. (in Japanese)

13 Jacobson, M., "Quantifying Membrane Wrinkle Behavior Due to In-Plane Shear," Master Dissertation, International Space University, Strasbourg, France, 2003.

14 Bucalem, M.L. and Bathe, K.J., "Higher-order MITC General Shell Elements," International Journal for Numerical Methods in Engineering, Vol. 36, 1993, pp. 3729-3754.

${ }^{15}$ Cerda, E., Ravi-Chandar, K., Mahadevan, L., "Thin Films: Wrinkling of An Elastic Sheet Under Tension," Brief Communications in Nature, Vol. 419, 2002, pp. 579-580.

${ }^{16}$ Iwasa, T., Natori, M.C., and Higuchi, K., "Comparative Study on Bifurcation Theory and Tension Field Theory for Wrinkling Analysis," Journal of Structural Engineering, Vol. 49B, 2003, pp. 319-326. (in Japanese)

17 Johnston, J. D., "Finite Element Analysis of Wrinkled Membrane Structures for Sunshield Applications," 43rd AIAA/ASME/ASCE/AHS/ASC Structures, Structural Dynamics and Materials Conference Proceedings, AIAA-20021456, 2002.

${ }^{18}$ Johnston, J. D., Blandino, J., Black, J., Pappa, R., "Structural Analysis and Testing of a Subscale Sunshield Membrane Layer," 44th AIAA/ASME/ASCE/AHS/ASC Structures, Structural Dynamics and Materials Conference Proceedings, AIAA-2003-1742, 2003.

${ }^{19}$ Miyamura, T., "Wrinkling on Stretched Circular Membrane Under In-plane Torsion: Bifurcation Analyses and Experiments," Engineering Structures, Vol. 23, 2000, pp. 1407-1425.

${ }^{20}$ Nakoshino, K., "Efficient Modification Scheme of Stress-Strain Tensor for Finite Element Analysis of Wrinkled Membranes," 44th AIAA/ASME/ASCE/AHS/ASC Structures, Structural Dynamics and Materials Conference Proceedings, AIAA-2003-1981, 2003.

21 Wong, Y.W. and Pellegrino, S., "Computation of Wrinkle Amplitudes in Thin Membranes," 43rd AIAA/ASME/ASCE/AHS/ASC Structures, Structural Dynamics and Materials Conference, AIAA-2002-1369, 2002.

${ }^{22} \mathrm{Su}, \mathrm{X}$., Abdi, F., Taleghani, B., and Blandino, J., "Wrinkling Analysis of a Kapton Square Membrane Under Tensile Loading," 44th AIAA/ASME/ASCE/AHS/ASC Structures, Structural Dynamics and Materials Conference Proceedings, AlAA-2003-1985, 2003. 